\title{
Activity-Based Probe for Ratiometric Fluorescence Imaging of Caspase-3 in Living Cells
}

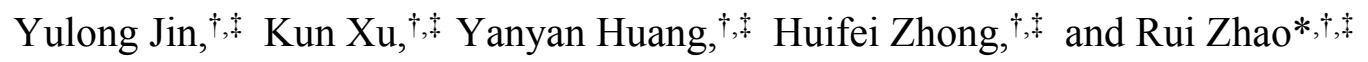

${ }^{\dagger}$ Beijing National Laboratory for Molecular Sciences, CAS Key Laboratory of Analytical Chemistry for Living Biosystems, CAS Research/Education Center for Excellence in Molecular Sciences, Institute of Chemistry, Chinese Academy of Sciences, Beijing, 100190, China

${ }^{*}$ School of Chemistry, University of Chinese Academy of Sciences, Beijing, 100049, China

*Corresponding Author

E-mail: zhaorui@iccas.ac.cn.

\section{Table of Contents}

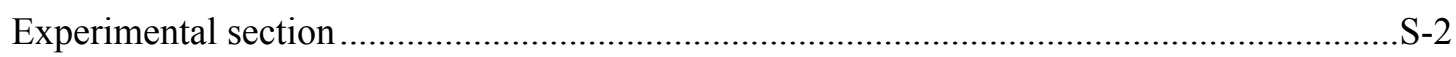

Figure S1. HPLC and ESI-MS analysis of the probe .............................................................

Figure S2. MALDI-FTICR mass spectrum of the probe ………………………….............

Figure S3. HPLC and ESI-MS analysis of the enzyme reaction............................................. -5

Figure S4. Fluorescence emission spectra of the probe after reaction with caspase-3 ...........S-5

Figure S5. Lineweaver-Burk plot for the enzyme-catalyzed reaction ...................................S-5

Table S1. Kinetic data of caspase-3 for Ac-DEVD-AFC and Ac-Tat-DEVD-CV ..................S-6

Figure S6. Caspase-3 inhibitors screening assay ………………...................................... -6

Figure S7. Viability of HeLa cells after incubation with the probe ........................................

Figure S8. HPLC and ESI-MS characterization of the control probe Ac-DEVD-CV .............S-6

Figure S9.Cell imaging study of the control probe Ac-DEVD-CV ………............................ -7

Figure S10. Cell imaging-costaining with LysoTracker ........................................................

Figure S11. Cell imaging-costaining with MitoTracker ..........................................................

Figure S12. Cell imaging-costaining with ER-Tracker......................................................

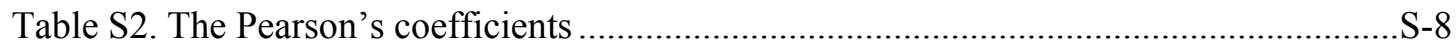




\section{Experimental section.}

\section{Materials and Apparatus.}

O-(7-Azabenzotriazol-1-yl)- $N, N, N^{\prime}, N^{\prime}$-tetramethyluroniumhexafluorophosphate (HATU), 2-(1H-Benzotriazole-1-yl)-1,1,3,3-tetramethyluronium hexafluorophosphate (HBTU) and Wang resin (1\% DVB, 200-400 mesh, $1.1 \mathrm{mmol} \mathrm{OH} / \mathrm{g}$ ) were all purchased from GL Biochem (Shanghai, China). 1-Hydroxybenzotriazole hydrate $\left(\mathrm{HOBT} \cdot \mathrm{H}_{2} \mathrm{O}\right)$ was obtained from Siam (Chicago, USA). Fmoc-L-Asp-OAll, Fmoc-L-Asp(OtBu)-OH, Fmoc-L-Val-OH, Fmoc-L-Glu(OtBu)-OH, Fmoc-Gly-OH, Fmoc-D-Arg(Pbf)-OH, Fmoc-D-Orn(Trt)-OH and Fmoc-D-Lys-OH were all purchased from GL Biochem (Shanghai, China) and Advanced ChemTech (Louisville, USA). Cresyl violet acetate and $\operatorname{Pd}\left(\mathrm{Ph}_{3} \mathrm{P}\right)_{4}$ were from J\&K Scientific Ltd. (Beijing, China). N,N'Diisopropylcarbodiimide (DIC), 4-(Dimethylamino)pyridine (DMAP) and sodium diethyldithiocarbamate trihydrate was purchased from Shanghai Aladdin Bio-Chem Technology Co., LTD (Shanghai, China). Caspase-3 inhibitor 5-[(S)-(+)-2(methoxymethyl)pyrrolidino]sulfonylisatin and Ac-DEVD-AFC were purchased from APExBIO Technology (Boston, USA). Recombinant human Caspase-3 and recombinant human Caspase-7 were both purchased from Sino Biological Inc. (Beijing, China). Recombinant human Caspase-6 were purchased from Abcam (Cambridge, UK). LysoTracker Deep Red, MitoTracker Deep Red and ER-Tracker Blue-White were all purchased from Thermo Fisher Scientific (Waltham, USA). 1,4Piperazinediethanesulfonic acid 3-[(3-Cholamidopropyl) dimethylammonium]-1-propanesulfonate (CHAPS), 3-(4,5-Dimethylthiazol-2-yl)2,5-diphenyltetrazolium bromide (MTT), cysteine, glutathione, glycine, glucose, vitamin $\mathrm{C}$, lysozyme, trypsin, BSA, pepsin, cytochrome $\mathrm{C}$ were all purchased from Sigma-Aldrich (St. Louis, USA). Staurosporine (STS), acetonitrile (ACN), methanol $(\mathrm{MeOH})$, trifluoroacetic acid (TFA) and triisoproyl silane (TIS) were purchased from Fisher Scientific (Hampton, USA) or Sigma-Aldrich. Dimethylformamide (DMF), dichloromethane (DCM), $\mathrm{CHCl}_{3}$, dimethyl sulfoxide (DMSO), piperidine, 4methylmorpholine (NMM), ethylenediaminetetraacetic acid (EDTA), NaCl, sucrose, acetic anhydride, pyridine, $N, N$ '-diisopropylethylamine (DIPEA), acetic acid, $\mathrm{HCl}$ and diethyl ether were all purchased from Beijing Chemical Works (Beijing, China). Dulbecco's modified Eagle medium (DMEM) and fetal bovine serum (FBS) were purchased from Invitrogen (California, USA). Ultrapure water from a Milli Q water purification system (Burlington, USA) was used throughout. Other materials used for synthesis were commercially available and used as received. All solvents were purified and dried following standard procedures unless otherwise stated.

Fluorescence measurements were made on a Hitachi F-4600 Fluorescence Spectrophotometer (Tokyo, Japan). UV-Vis adsorption spectra were recorded on a Shimadzu UV-1800 spectrometer (Kyoto, Japan). Relative quantum yield $(\Phi)$ of the probe before and after reaction with caspase- 3 was determined by using CV $(\Phi=0.54$ in methanol) as a standard. Enzymatic assay in solutions were made on a microplate reader (Molecular Devices SpectraMax M2e). 
The HPLC-ESI-IT-MS analysis was performed on an UltiMate 3000 UHPLC system equipped with a diode-array detector and connect to an LCQ Fleet mass spectrometer (ThermoFisher, Waltham, USA). The chromatographic column was a Welch Ultimate XB-C18 column, with $1.8 \mu \mathrm{m}$ particle (column dimensions $2.1 \times 100 \mathrm{~mm}$ ). Highresolution mass spectra were recorded on a Solarix 9.4T AS FTICR MS equipped with a MALDI source (Bruker Daltonics, Bremen, Germany). The cells were imaged by fluorescence microscopy (FV 1000-IX81 confocal microscope).

\section{Western blotting assay}

For the analysis of the expression level of caspase-3 in cells, western blotting assay was performed. To induce cell apoptosis, STS solution ( $1 \mu \mathrm{M}$ in DMEM) was incubated with HeLa cells for $1 \mathrm{~h}, 3 \mathrm{~h}$, or $4 \mathrm{~h}$ respectively. When caspase- 3 inhibitor was used in the assay, HeLa cells were treated with caspase- 3 inhibitor $(10 \mu \mathrm{M})$ and STS $(1 \mu \mathrm{M})$ in DMEM for $3 \mathrm{~h}$. Then, untreated or STS-treated HeLa cells were washed with PBS for 3 times. After cell lysis and centrifuge, the cell lysates were collected for protein content determination using BCA assay. Proteins in cell lysates were then separated by SDSPAGE and transferred to PVDF membrane. The membrane was blocked with QuickBlock $^{\mathrm{TM}}$ blocking buffer (Beyotime, China) for $15 \mathrm{~min}$, followed by the incubation with rabbit anti-CASP3 antibody (Sino Biological Inc., dilution 1:500) and mouse anti-actin antibody (Santa Cruz Biotechnology Inc., dilution 1:2000) at $4{ }^{\circ} \mathrm{C}$ overnight. The membrane was then washed with TBSTw (Beyotime, China) 5 times and incubated with horseradish peroxidase-conjugated goat anti-rabbit antibody (Transgenbiotech, dilution 1:1000) or goat anti-mouse antibodies (Transgenbiotech, dilution 1:5000) at room temperature for $1 \mathrm{~h}$. After washed with TBSTw, the membrane was visualized with chemiluminescence reagent (Biotanon, China). Quantitative analysis of protein expression levels was realized using Image-J software.

\section{Caspase-3 inhibitors screening assay}

HeLa cells were treated with STS solution ( $1 \mu \mathrm{M}$ in DMEM) for $3 \mathrm{~h}$. The cells were then washed with PBS for 3 times. After cell lysis and centrifuge, the cell lysates were collected and different concentrations of inhibitor candidates were added into the cell lysate. Then Ac-Tat-DEVD-CV $(10 \mu \mathrm{M}$, final concentration) was added to the solution with the incubation for $30 \mathrm{~min}$. Fluorescence data were collected with $\lambda_{\mathrm{ex}}=528 \mathrm{~nm}$, and $\lambda_{\mathrm{em}}=582 \mathrm{~nm}, 628 \mathrm{~nm}$; the fluorescent ratio $\left(I_{628} / I_{582}\right)$ was then calculated. The inhibition ratio was calculated by the equation: Inhibition Ratio $=\left(R-R_{\mathrm{i}}\right) /\left(R-R_{\mathrm{b}}\right) \times$ $100 \%$, where $R_{\mathrm{i}}$ and $R$ are the ratio value of the reaction solution with and without inhibitor, respectively; $R_{\mathrm{b}}$ is the ratio value of the probe in assay buffer (blank).

\section{Co-staining assay}

For co-staining assay, HeLa cells were first treated with organelle-specific trackers, followed by the incubation with probe. After washed with PBS for 3 times, HeLa cells were observed with CLSM. MitoTracker Deep Red and LysoTracker Deep Red were both excited by a $635 \mathrm{~nm}$ laser and collected with a band-pass filter within the range of 650-750 nm. ER-Tracker Blue-White was excited by a $405 \mathrm{~nm}$ laser and collected with 
a band-pass filter within the range of 450-550 nm. Probe was excited by a $515 \mathrm{~nm}$ laser and collected in two channels, 530-595 nm (Green Channel) and 615-670 nm (Red Channel).
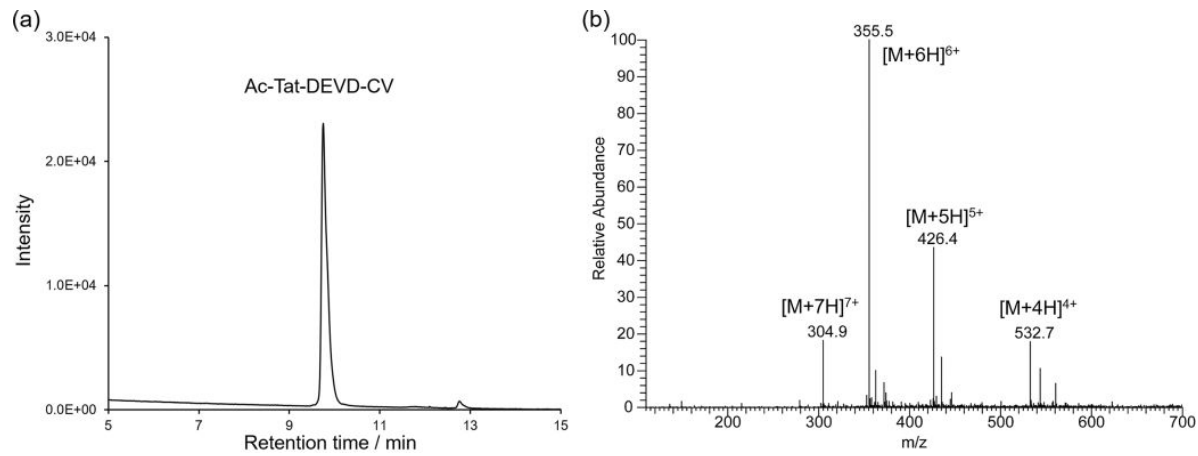

Figure S1. (a) HPLC analysis of Ac-Tat-DEVD-CV on a C18 column. Gradient: 0-15min, 10\%B$60 \% \mathrm{~B}\left(\mathrm{~A}: 0.1 \% \mathrm{TFA} / \mathrm{H}_{2} \mathrm{O}, \mathrm{B}: 0.1 \% \mathrm{TFA} / \mathrm{CH}_{3} \mathrm{CN}\right.$ ). (b) ESI-IT mass spectrum of Ac-Tat-DEVD$\mathrm{CV}$ operated in positive ion mode.
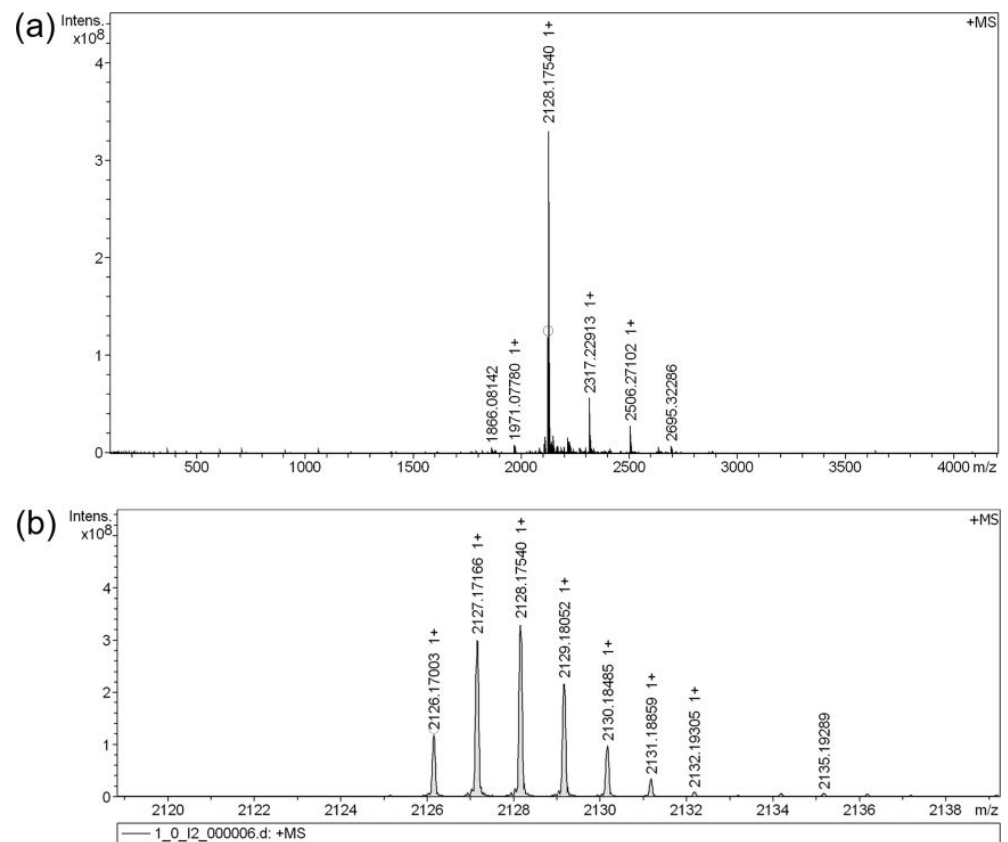

Figure S2. MALDI-FTICR mass spectrum of Ac-Tat-DEVD-CV operated in positive ion mode: $\mathrm{m} / \mathrm{z}[\mathrm{M}+\mathrm{H}]^{+}$calcd: 2126.1703; found: 2126.1700. (a) Positive ion mode, full scan. (b) Isotopic ion peaks. 

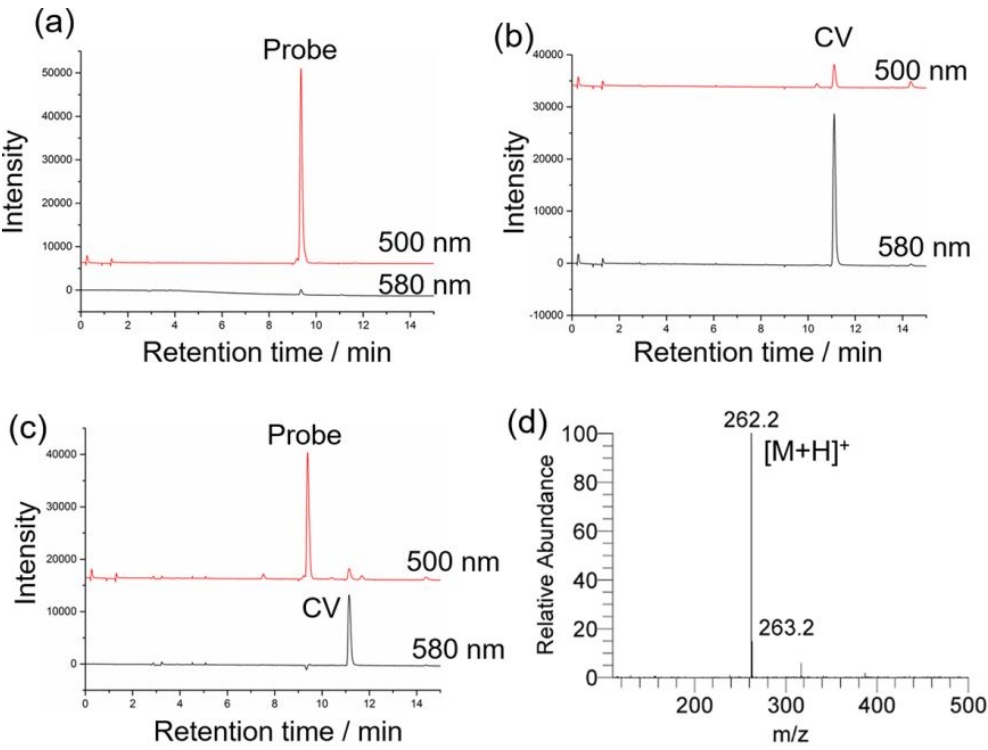

Figure S3. HPLC and ESI-MS analysis of the enzyme reaction between caspase-3 and probe. Red line: monitored at $500 \mathrm{~nm}$ (characteristic absorption wavelength of Probe). Black line: monitored at $580 \mathrm{~nm}$ (characteristic absorption of CV). (a) probe only; (b) CV standard; (c) probe+caspase-3; (d) mass spectrum of the new peak appeared in figure S3c.

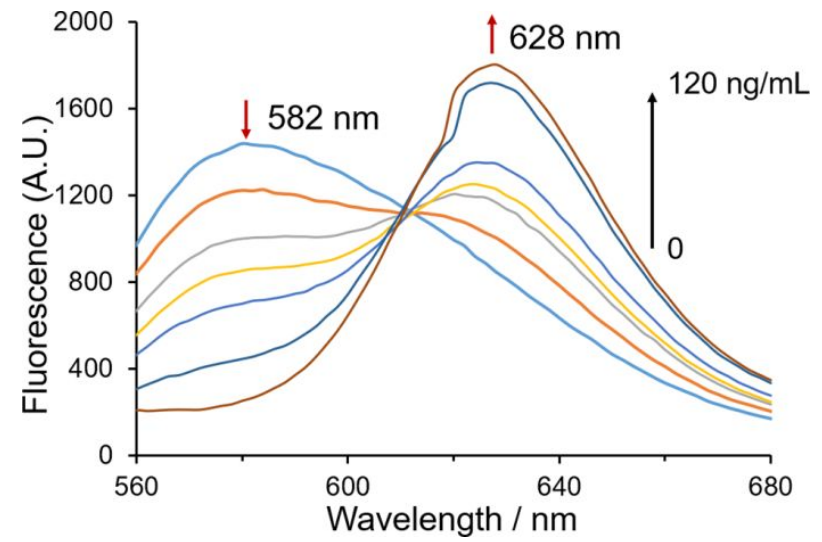

Figure S4. Fluorescence emission spectra of Ac-Tat-DEVD-CV $(10 \mu \mathrm{M})$ with different concentration of caspase-3 (from bottom to top): $0,10,20,30,40,80,120 \mathrm{ng} / \mathrm{mL}$.
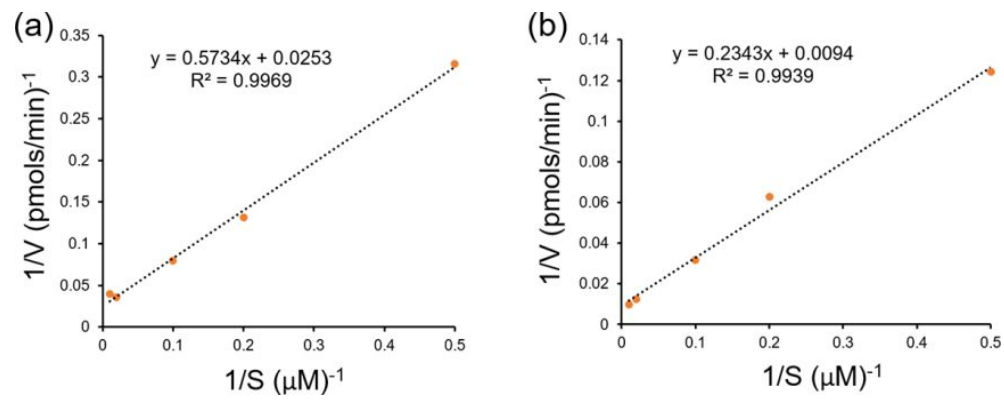

Figure S5. Lineweaver-Burk plot for the enzyme-catalyzed reaction. The Michaelis- Menten equation was described as: $V_{0}=k_{\text {cat }}[\mathrm{E}]_{0}[\mathrm{~S}] /\left(K_{\mathrm{M}}+[\mathrm{S}]\right)$, where $V_{0}$ is the initial reaction rate, $k_{\text {cat }}$ is the turnover number, $[\mathrm{E}]_{0}$ is the enzyme concentration (caspase-3), $[\mathrm{S}]$ is the probe concentration, and $K_{\mathrm{M}}$ is the Michaelis constant. (a)Ac-DEVD-AFC; (b)Ac-Tat-DEVD-CV. The concentration of the product $(\mathrm{CV})$ formed during the catalytic reaction was quantified by fluorescence ratio change. 
Table S1. Kinetic data of caspase-3 for Ac-DEVD-AFC and Ac-Tat-DEVD-CV.

\begin{tabular}{|c|c|c|c|}
\hline \multirow{2}{*}{ Probe } & \multicolumn{3}{|c|}{ Caspase-3 } \\
\cline { 2 - 4 } & $K_{\mathrm{M}}(\mu \mathrm{M})$ & $k_{c a t}\left(\mathrm{~S}^{-1}\right)$ & $k_{c a t} / K_{\mathrm{M}}\left(\mu \mathrm{M}^{-1} \mathrm{~S}^{-1}\right)$ \\
\hline Ac-DEVD-AFC & 22.6 & 3.9 & 0.17 \\
\hline Ac-Tat-DEVD-CV & 24.9 & 10.6 & 0.43 \\
\hline
\end{tabular}
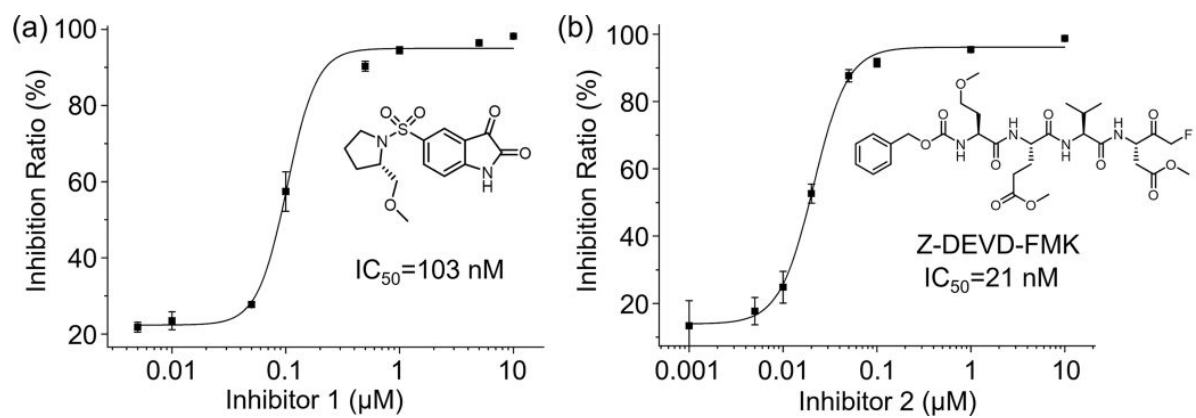

Figure S6. Caspase-3 inhibitors screening assay with Ac-Tat-DEVD-CV as the sensing probe.

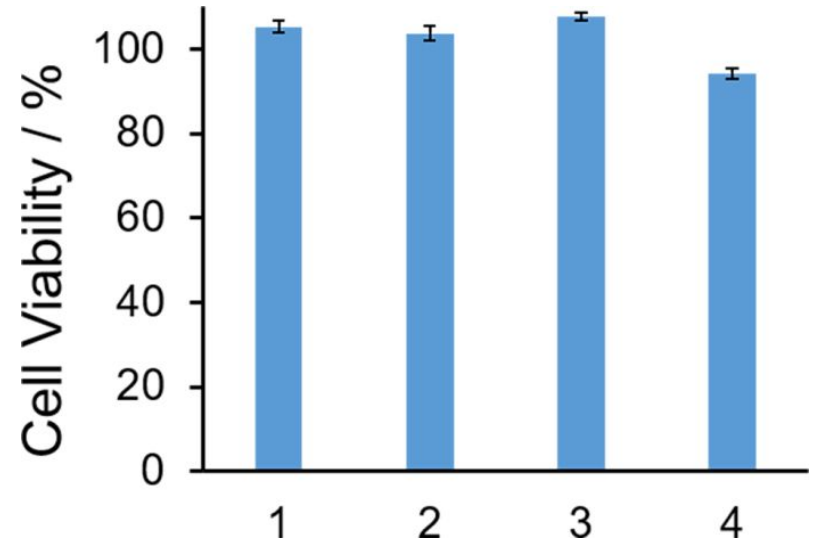

Figure S7. Viability of HeLa cells after incubation with Ac-Tat-DEVD-CV at two concentrations, $1 \mu \mathrm{M}(1,3)$ and $10 \mu \mathrm{M}(2,4)$ for 24 hours based on MTT assays. For 1 and 2, probe was incubated with cells for 2 hours, then discarded the probe solution and added DMEM solution for further incubation. For 3 and 4, probe was incubated with cells for 24 hours. All tests were conducted in triplicate.
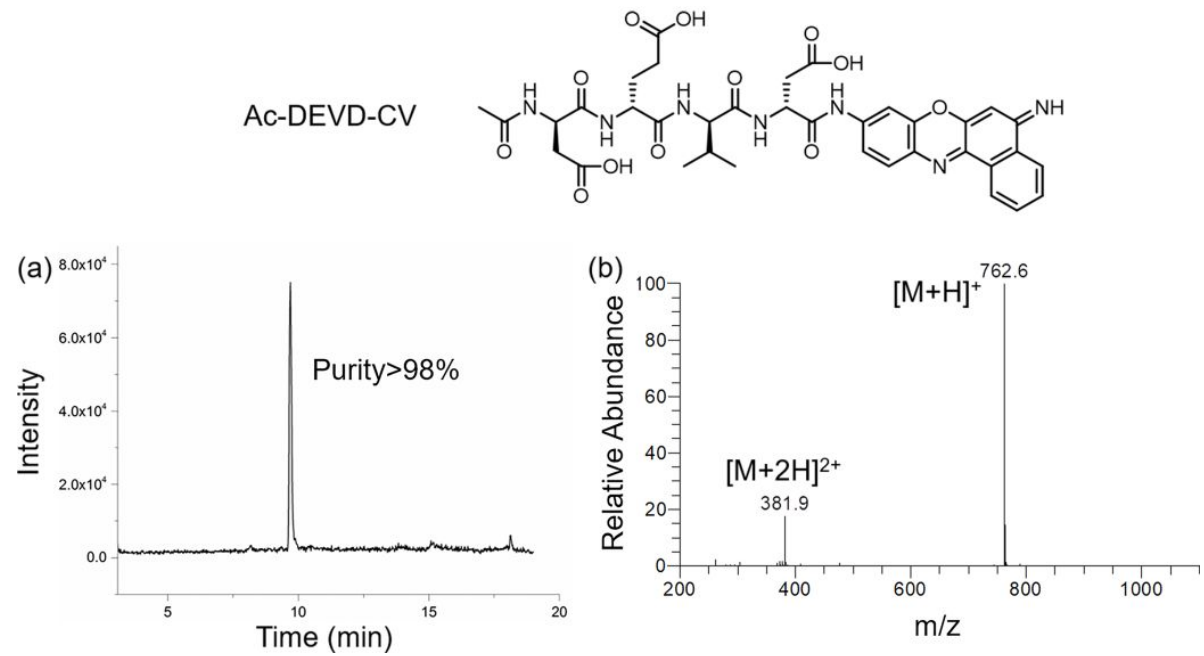

Figure S8. The characterization of the control probe, Ac-DEVD-CV. (a) HPLC analysis of Ac- 
DEVD-CV on a C18 column. (b) ESI-IT mass spectrum of Ac-DEVD-CV operated in positive ion mode.

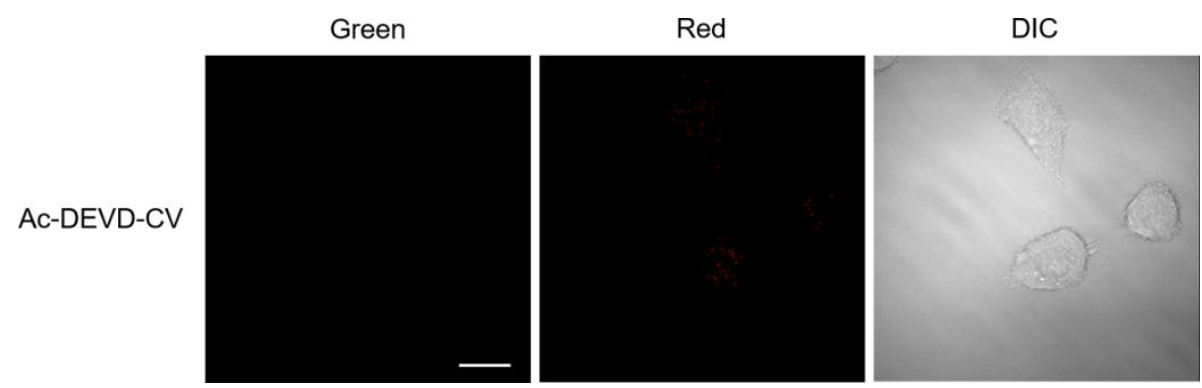

Figure S9. Fluorescence microscope images. HeLa cells were incubated with Ac-DEVD-CV (10 $\mu \mathrm{M}, 200 \mu \mathrm{L})$ for $2 \mathrm{~h}$ at $37^{\circ} \mathrm{C}$. All images share the same scale bar $(20 \mu \mathrm{m})$.

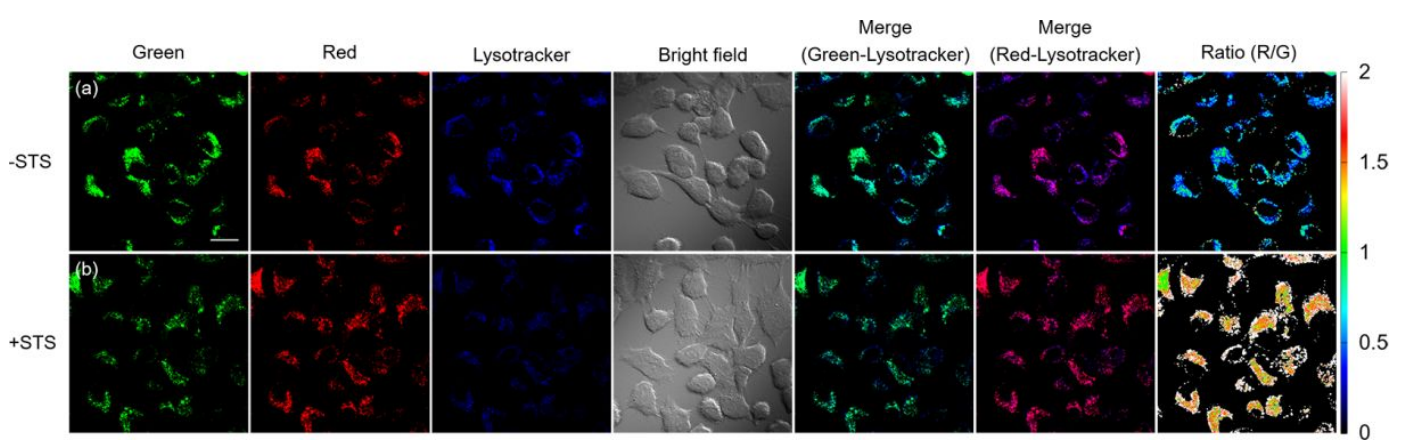

Figure S10. Fluorescence microscope images. Row (a): HeLa cells treated with LysoTracker Deep Red $(50 \mathrm{nM})$ and probe $(10 \mu \mathrm{M})$; Row (b): HeLa cells treated with LysoTracker Deep Red (50 nM), probe $(10 \mu \mathrm{M})$ and STS $(1 \mu \mathrm{M})$. The color strip represented the pseudo color change with different ratio values. All images share the same scale bar $(20 \mu \mathrm{m})$.

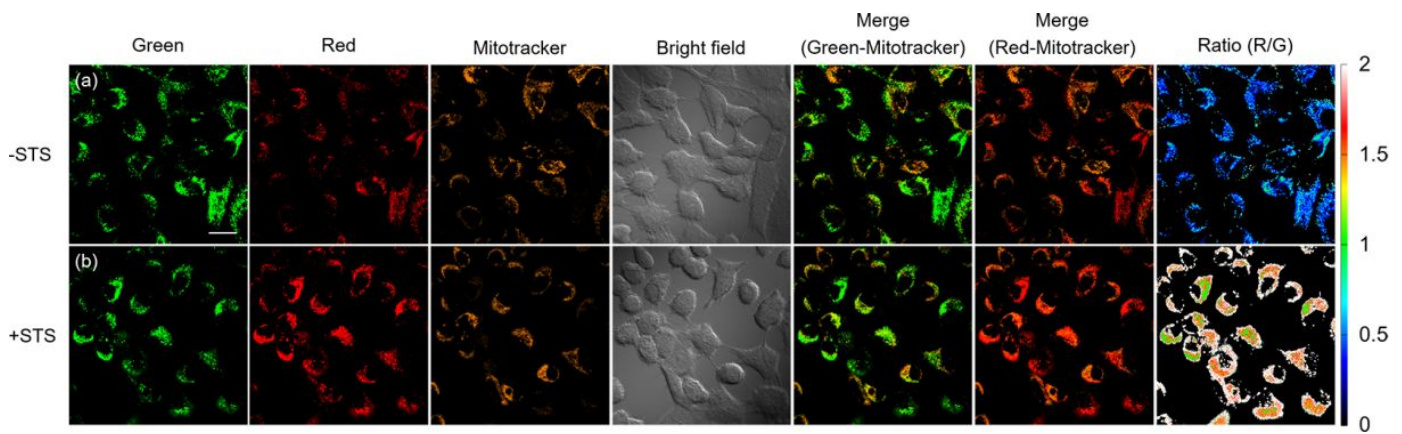

Figure S11. Fluorescence microscope images. Row (a): HeLa cells treated with MitoTracker Deep Red $(200 \mathrm{nM})$ and probe $(10 \mu \mathrm{M})$; Row (b): HeLa cells treated with MitoTracker Deep Red (200 $\mathrm{nM})$, probe $(10 \mu \mathrm{M})$ and STS $(1 \mu \mathrm{M})$. The color strip represented the pseudo color change with different ratio values. All images share the same scale bar $(20 \mu \mathrm{m})$. 


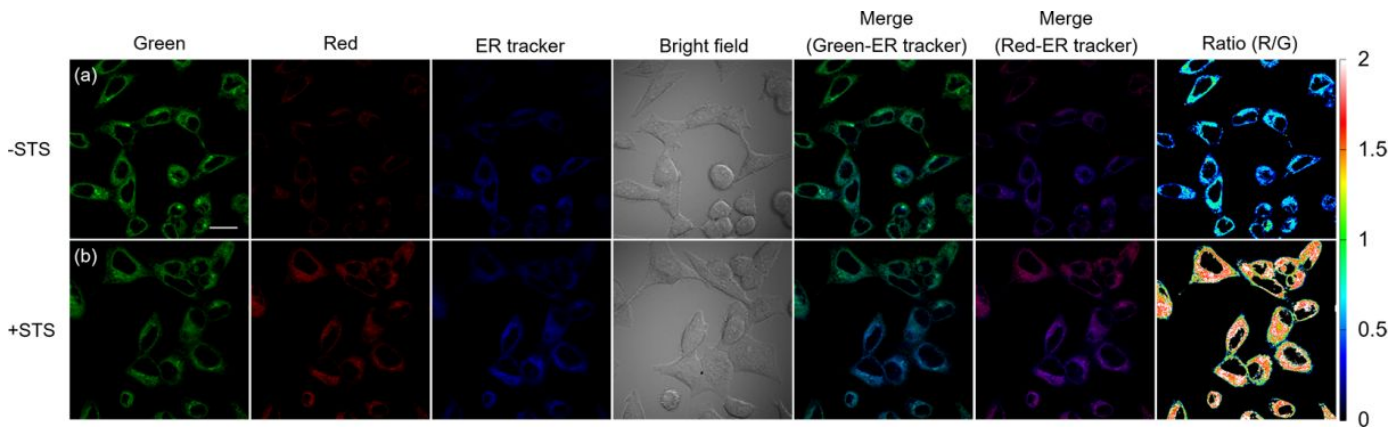

Figure S12. Fluorescence microscope images. Row (a): HeLa cells treated with ER-Tracker BlueWhite (200 nM) and probe $(10 \mu \mathrm{M})$; Row (b): HeLa cells treated with ER-Tracker Blue-White (200 $\mathrm{nM})$, probe $(10 \mu \mathrm{M})$ and STS $(1 \mu \mathrm{M})$. The color strip represented the pseudo color change with different ratio values. All images share the same scale bar $(20 \mu \mathrm{m})$.

Table S2. The Pearson's coefficient of the probe's Green and Red channel with organelle-specific trackers in untreated or STS-treated HeLa cells.

\begin{tabular}{|c|c|c|c|c|c|c|}
\hline \multirow{2}{*}{ Pearson's coefficient } & \multicolumn{2}{|c|}{ LysoTracker } & \multicolumn{2}{c|}{ MitoTracker } & \multicolumn{2}{c|}{ ER-Tracker } \\
\cline { 2 - 7 } & Untreated & STS-treated & Untreated & STS-treated & Untreated & STS-treated \\
\hline Green Channel & 0.73 & 0.65 & 0.26 & 0.25 & 0.70 & 0.68 \\
\hline Red Channel & 0.70 & 0.69 & 0.21 & 0.24 & 0.64 & 0.85 \\
\hline
\end{tabular}

